\title{
Investigating Ultrasonic Diffraction Grating Spectroscopy and Reflection Techniques for Characterizing Slurry Properties
}

Project Number: 81889

Lead Principal Investigator:

Margaret S. Greenwood

Pacific Northwest National Laboratory

Richland, WA 99352

509-375-6801

Margaret.greenwood@pnl.gov

Co-Investigator:

Leonard J. Bond

Pacific Northwest National Laboratory

Richland, WA 99352

509-375-4486

Leonard.bond@pnl.gov

Co-Investigator:

Lloyd Burgess

University of Washington

Seattle, WA 98195

206-543-0579

lloyd@cpac.washington.edu

Co-Investigator:

Anatol Brodsky

University of Washington

Seattle, WA 98195

206-543-1676

brodsky@cpac.washington.edu

Graduate Student:

Mazen Lee Hamad

University of Washington

Seattle, WA 98195

mhamad@u.washingtonedu

\section{Research Objective}

The objectives of the project are to investigate the use of 1) ultrasonic diffraction grating spectroscopy (UDGS) to measure the velocity of sound in a liquid or slurry and the particle size of a slurry and 2) shear wave reflection techniques to measure the viscosity of a slurry. 


\section{Research Progress and Implications}

This report summarizes work after two years of a three-year project. The ultrasonic diffraction grating is formed by machining triangular grooves, several hundred microns apart, on a stainless steel (SS) surface. The grating surface is in contact with the liquid or slurry. The ultrasonic beam, traveling in stainless steel, strikes the grating and produces a transmitted $m=1$ beam in the liquid. The angle of this beam in the liquid increases with decreasing frequency and the critical frequency $\mathrm{F}_{\mathrm{CR}}$ occurs when the angle is $90^{\circ}$. At frequencies below $\mathrm{F}_{\mathrm{CR}}$, this $\mathrm{m}=1$ wave does not exist and its energy is shared with other types of waves. The signal of the reflected $\mathrm{m}=0$ wave is observed and an increase is observed at $\mathrm{F}_{\mathrm{CR}}$. This information yields the velocity of sound in the liquid and the particle size. At the critical frequency, the waves become evanescent, as shown in Fig. 1. The possibility of UDGS to measure particle size is due to the penetration of the evanescent wave into the slurry. As the wave interacts with the particle, some attenuation of the signal occurs. As a result, the signal measured by the receive transducer is expected to decrease in amplitude. Since the attenuation is dependent upon particle size, an algorithm can be developed to determine particle size.

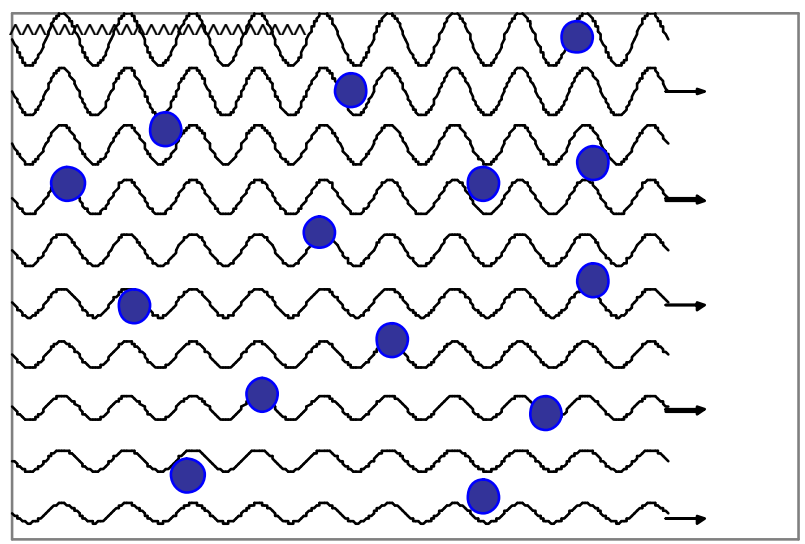

Fig. 1. Schematic diagram of an evanescent wave, produced by the diffraction grating (upper left), interacting with particles in a slurry.

In the first set of measurements reported in the Annual Report for 2002, a peak occurred in the data for the reflected $\mathrm{m}=0$ wave. The peak was observed for a variety of incident angles and for gratings with two different spacings, 200 microns and 300 microns. However, the height of the peak was not large. During this year, the experimental apparatus was changed to increase the peak height. One possibility was to increase the size of the transducers and a second, to explore changes in the grating design. Both were explored. The results showed that increasing the transducer size, from 0.25 in. diameter to 0.5 inches square, does increase the peak height and reduces the width of the peak. The first set of gratings were machined with a $120^{\circ}$ included angle for the triangular shaped grooves. A grating with a $90^{\circ}$ included angle and a spacing of 300 microns was fabricated. The results show that the peak width was increased significantly, which was not desirable. The conclusion is that the $120^{\circ}$ included angle is the better choice. Fig. 2 shows the data obtained for water and several sugar water solutions. The peak height is increased significantly and the width is reduced to $0.5 \mathrm{MHz}$ in this figure.

The peaks in Fig. 2 raise questions about the mechanism when the $m=1$ transmitted longitudinal wave becomes evanescent: Why doesn't the voltage remain at the peak value at frequencies below the critical frequency? To answer this question data were obtained for the $\mathrm{m}=0$ longitudinal wave in the liquid and for the $m=0$ reflected shear wave (using shear wave transducers cemented to the stainless steel grating). The conclusion is that the energy initially contained in the $\mathrm{m}=1$ transmitted longitudinal wave is transferred primarily to the $\mathrm{m}=0$ transmitted longitudinal wave after the transition. However, 
during the transition, energy is shared primarily with the reflected $m=0$ shear wave and the $m=0$ transmitted longitudinal wave. The critical frequency occurs at the maximum value of the peak
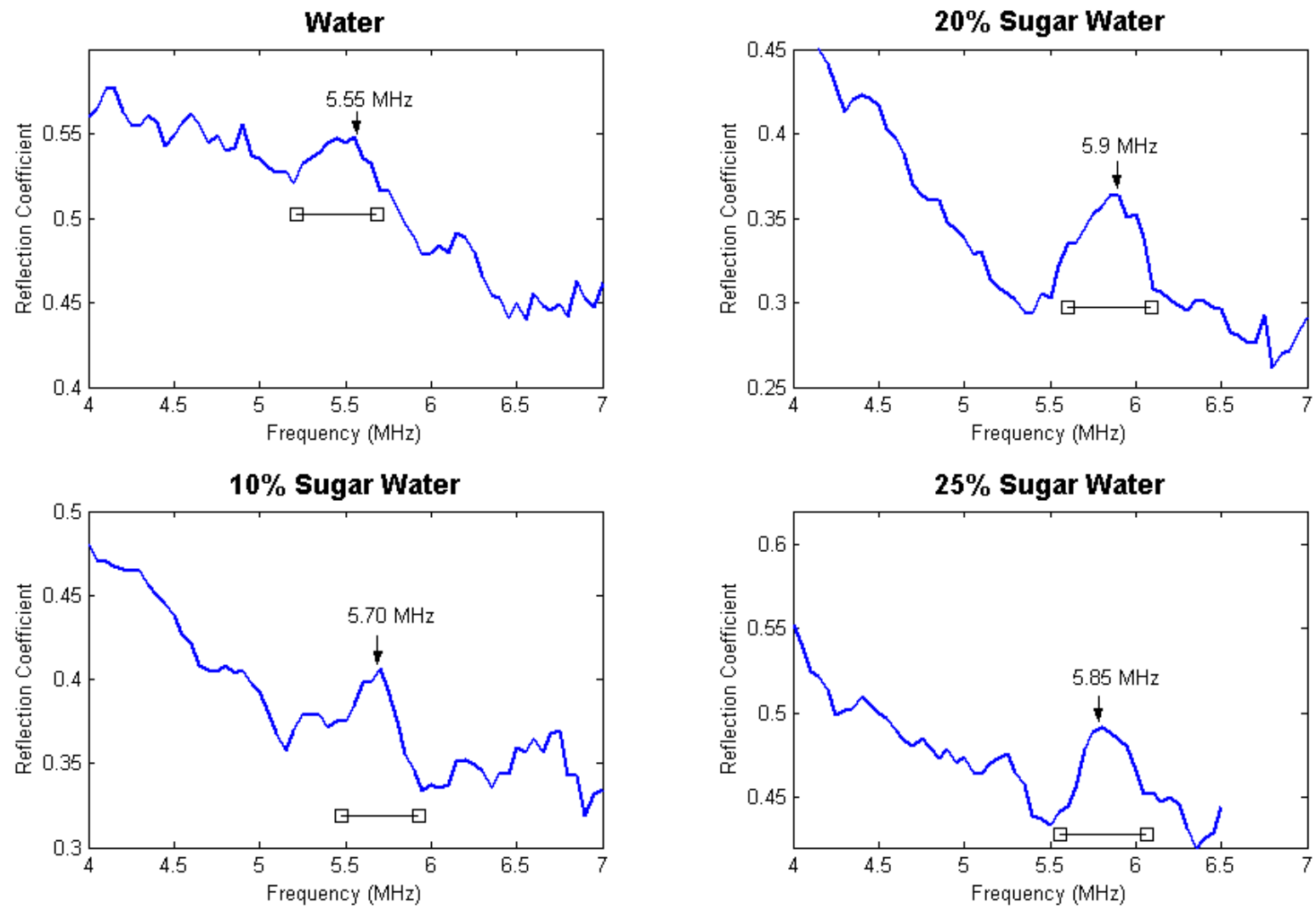

Fig. 2. Experimental data for the $m=0$ reflected longitudinal wave for water, $10 \%$ sugar water, $20 \%$ sugar water, and $25 \%$ sugar water. The frequency of the maximum amplitude is shown for each liquid.

The immersion chamber, in which the stainless steel grating is placed, is mounted upon a turntable that is motorized and computer-controlled. This permits observation of the $\mathrm{m}=1$ longitudinal wave in the liquid before it reaches the $90^{\circ}$ angle and becomes evanescent. Scans over an angular range and scans over a frequency range are helpful in interpretation of the data.

During June 2003, an apparatus for the measurement of particle size was fabricated. It consists of a stainless steel grating, having a grating spacing of 240 microns, with 3/8 diameter transducers cemented to the stainless steel. For evaluation of the transducer response, data were obtained first when the front surface was smooth. Then the grating was machined onto the flat surface. The first step is to obtain data for liquids and compare the critical frequencies with those expected theoretically. That step is nearing completion at the time of this writing and the analysis is underway.

During the last year, the main objective of the theoretical work at the University of Washington has been to develop a method to calculate the ultrasonic dissipation parameters from Ultrasonic Diffraction Grating Spectroscopy (UDGS) experimental data. Such parameters will allow us to estimate the general particle characteristics in the planned experiments with heterogeneous slurries. In the present experiments with homogeneous liquid samples, the main dissipation effect is related to the viscosity of 
the liquid samples. The capability of simultaneously measuring the viscosity along with the velocity of sound is of considerable practical importance.

We have developed a data analysis program that allows us to calculate the viscosity coefficients from the frequencies of the UDGS threshold singularity positions and the heights of the extrema in the first derivatives of the signal over frequency. It has been determined that the distance between the frequency positions of the maxima and the minima in the derivative signals is proportional to the viscosity of the samples. The preliminary comparison of the theory and the existing experimental data confirms the trend expected from the theory.

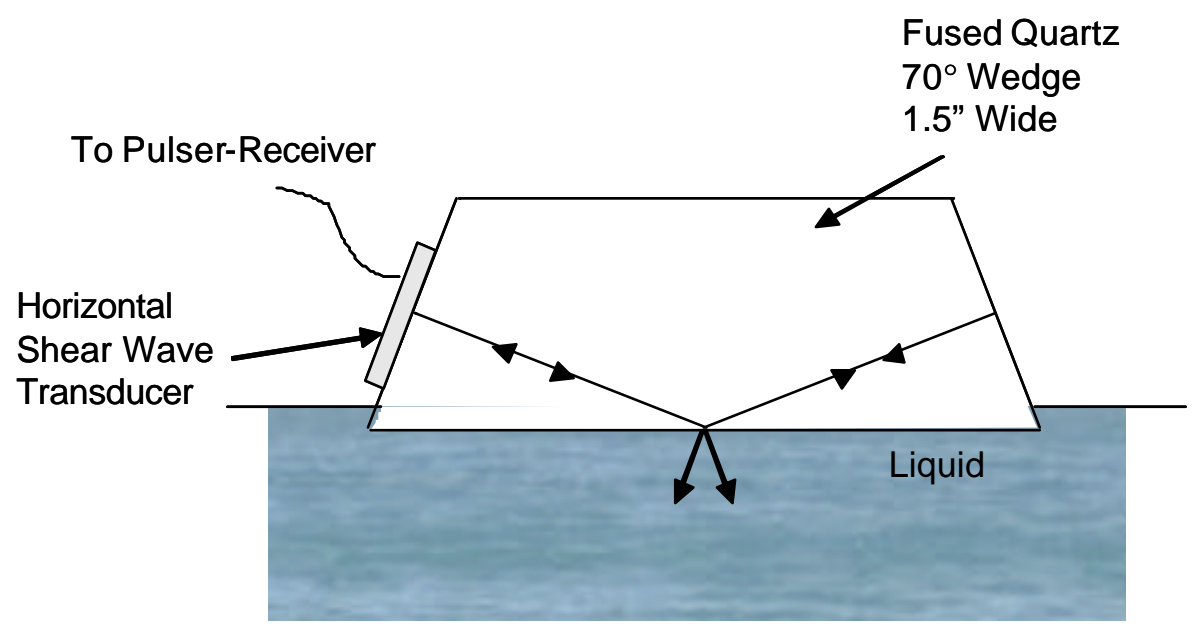

Fig. 3. Sensor for measurement of viscosity.

The second part of the EMSP project, as stated in the objectives, concerns reflection techniques to measure the viscosity of a liquid or slurry. In Fig. 3, a 7.5 MHz horizontal shear wave transducer is mounted on a $70^{\circ}$ fused quartz wedge. More than 20 echoes are recorded by the transducer as the ultrasound makes multiple reflections between the inclined surfaces. Each time the ultrasound strikes the quartz-liquid interface an amount, dependent upon the viscosity, is transmitted into the liquid. The multiple reflections amplify the effect and, by measuring the reflection coefficient, the viscosity can be determined by using water as a reference liquid. The results at $22 \mathrm{C}$ are shown in Table 1 . Considering that the viscosity measurement is quite temperature dependent, the results are in good agreement with the Handbook values at $20 \mathrm{C}$, especially for sugar water concentrations greater than $30 \%$.

A fused quartz wedge with a $14 \mathrm{MHz}$ transducer has just been fabricated and data will be obtained during this summer. It is expected to have greater sensitivity.

The quartz wedge can be part of pipeline wall so that a computer-controlled sensor can be developed to measure viscosity on-line and in real time for DOE applications. Also, a sensor for insertion into a tank can also be developed.

Justus Adamson, a junior physics major, is working on the EMSP project this summer. Data are being obtained using the newly-fabricated ultrasonic diffraction grating device and the quartz wedges for viscosity measurements. The results of these measurements will be submitted for publication in the autumn, 2003 and presented at the American Chemical Society National Meeting in September, 2000 
Table 1. Results of measurement of viscosity and comparison with accepted values.

\begin{tabular}{||c|c|c|c||}
\hline $\begin{array}{c}\text { Sugar Water } \\
\text { Concentration by } \\
\text { Weight Percentage }\end{array}$ & $\begin{array}{c}\text { Density } \\
\left(\mathbf{g} / \mathbf{c m}^{\mathbf{3}}\right)\end{array}$ & $\begin{array}{c}\text { Experimental } \\
\text { Viscosity at } \\
\text { 22 C }(\mathbf{c P})\end{array}$ & $\begin{array}{c}\text { Handbook } \\
\text { Value } \\
\text { Viscosity at } \\
\mathbf{2 0} \mathbf{C}(\mathbf{c P})\end{array}$ \\
\hline 5 & 1.016 & 4.4 & 1.14 \\
\hline 10 & 1.037 & 2.9 & 1.33 \\
\hline 15 & 1.058 & 4.6 & 1.59 \\
\hline 20 & 1.080 & 5.2 & 1.94 \\
\hline 25 & 1.103 & 7.9 & 2.44 \\
\hline 30 & 1.127 & 4.5 & 3.18 \\
\hline 35 & 1.151 & 3.0 & 4.33 \\
\hline 40 & 1.176 & 4.7 & 6.15 \\
\hline 45 & 1.203 & 6.9 & 9.43 \\
\hline 50 & 1.229 & 14.2 & 15.40 \\
\hline 55 & 1.258 & 24.8 & 28.34 \\
\hline 60 & 1.277 & 42.1 & 42.69 \\
\hline
\end{tabular}

\section{Planned Activities}

1. Continued development of the viscosity sensor for $14 \mathrm{MHz}$. Analysis of data to be completed by October 15, 2003.

2. Carry out measurements with particle size apparatus using three diameters of polystyrene spheres by November 2003.

3. Develop algorithm for particle sizing and compare with data. December 2003.

4. Obtain data over a large range of particle sizes. March 2004.

5. Refine measurement techniques for particle sizing so that it can be used for process control. July 2004.

\section{Information}

Publication:

M. S. Greenwood, A. M. Brodsky, L. W. Burgess, L. J. Bond, Using ultrasonic diffraction grating spectroscopy to characterize fluids and slurries, Review of Progress in Quantitative Nondestructive Evaluation, Volume 22B, 1637-1643 (2002).

Presentation at Ultrasonics International Conference 2003, Granada, Spain, July 1, 2003: "Ultrasonic diffraction grating spectroscopy and measurement of the particle size of a slurry." Paper will be published in journal Ultrasonics.

The presentation at the EMSP workshop held at Pacific Northwest National Laboratory on May 6-7, 2003, is available at www.pnl.gov/emsp under "presentations."

A U. S. Patent application has been filed for characterizing fluids using ultrasonic diffraction grating spectroscopy. 
Presentation at the American Chemical Society National Meeting, September 7-11,2003, in New York City in a symposium entitled "Environmental Management Science Program Symposium on Nuclear Waste Management. The manuscript will be published in a symposium book. 